\title{
A GENERALIZATION OF A THEOREM OF N. ITÔ ON $p$-GROUPS ${ }^{1}$
}

\author{
CHARLES HOBBY AND C. R. B. WRIGHT
}

We shall use the following notation: $G$ is a finite $p$-group; $\phi(G)$ is the Frattini subgroup of $G$; if $M$ and $N$ are subgroups of $G$, then $(M, N)$ is the subgroup of $G$ generated by the set of all expressions $m^{-1} n^{-1} m n$ for $m \in M$ and $n \in N ; M \subset N$ means $M$ is properly contained in $N$. The descending central series $G_{1}, G_{2}, \cdots, G_{n}$, of $G$ is defined recursively by $G_{1}=G$ and $G_{m+1}=\left(G, G_{m}\right)$.

N. Itô [2] has shown that if $H_{2} \subset G_{2}$ then $(H \phi(G))_{2} \subset G_{2}$. Our main result is the following generalization of Itô's theorem.

THEOREM 1. If $G$ is a finite p-group and $H$ a subgroup of $G$ such that $H_{n} \subset G_{n}$, then $(H \phi(G))_{n} \subset G_{n}$.

We first prove two lemmas, the second of which is of independent interest.

Lemma 1. Let $H$ be a subgroup of the p-group $G$. Then $\left(G, H_{n}\right)$ is contained in any normal subgroup of $G$ which contains $\left(G_{n}, H\right)$.

Proof. The lemma is certainly true if $n=1$, so suppose it is true for $n=k$ and all pairs $\{G, H\}$. It is known [1, Theorem 10.3.5] that $\left(G, H_{k+1}\right)=\left(G,\left(H_{k}, H\right)\right)$ is contained in any normal subgroup of $G$ which contains $\left(\left(G, H_{k}\right), H\right)$ and $\left((G, H), H_{k}\right)$. But $\left(G, H_{k}\right) \subseteq G_{k+1}$, and by the induction assumption $\left((G, H), H_{k}\right)$ is contained in any normal subgroup of $(G, H)$ which contains $\left((G, H)_{k}, H\right)$; hence $\left((G, H), H_{k}\right)$ is contained in any normal subgroup of $G$ which contains $\left((G, H)_{k}, H\right)$. Since $(G, H)_{k} \subseteq G_{2 k} \subseteq G_{k+1}$, it follows that $\left((G, H)_{k}, H\right)$ is contained in any normal subgroup of $G$ which contains $\left(G_{k+1}, H\right)$. Thus $\left(G,\left(H_{k}, H\right)\right)$ is contained in any normal subgroup of $G$ which contains $\left(G_{k+1}, H\right)$, and the lemma follows by induction.

Lemma 2. If $G$ is a finite p-group and $H$ a subgroup of $G$ such that $H_{n} \subset G_{n}$, then $H_{n} G_{n+1} \subset G_{n}$.

Proof. Let $G$ be a group of minimal order such that there exist a subgroup $K$ of $G$ and an integer $m$ for which the lemma is false, i.e., such that $K_{m} \subset G_{m}$ but $K_{m} G_{m+1}=G_{m}$. In $G$, let $H$ be a subgroup of

Presented to the Society April 23, 1960, under the title The lower central series of a p-group; received by the editors January 23, 1960.

1 This work was supported in part by the Office of Naval Research through contract MR 044-213. 
maximal order for which the lemma is false, and suppose that $n$ is an integer such that $H_{n} \subset G_{n}$ but $H_{n} G_{n+1}=G_{n}$.

Certainly $G_{n+1}$ contains a normal subgroup $N$ of $G$ such that $H_{n} N=G_{n}$, and if $M \subset N$ and $M$ is normal in $G$, then $H_{n} M \subset G_{n}$. If $N$ does not have order $p$ there exists a subgroup $M \subset N$ such that $[N: M]=p$ and $M$ is normal in $G$. Then $(H M / M)_{n}=H_{n} M / M$ $C G_{n} / M=(G / M)_{n}$, while $(H M / M)_{n}(G / M)_{n+1}=\left(H_{n} G_{n+1}\right) / M=G_{n} / M$ $=(G / M)_{n}$. Thus the lemma is false for $G / M$; hence $M=\langle 1\rangle$ since $G$ is a group of minimal order for which the lemma is false. It follows that the normal subgroup $N$ must have order $p$; hence $N$ is contained in the center of $G$. Since $H_{n} \subset G_{n}$ and $H_{n} N=G_{n}$, we have $G_{n}=H_{n} \times N$.

Let $K$ be the normalizer of $H$ in $G$. If $K=G$, then $H_{n}$, as a characteristic subgroup of the normal subgroup $H$, is normal in $G$. But then $\left(G / H_{n}\right)_{n+1}=H_{n} G_{n+1} / H_{n}=G_{n} / H_{n}=\left(G / H_{n}\right)_{n}$. Since $G / H_{n}$ is nilpotent, it follows that $\left(G / H_{n}\right)=\langle 1\rangle$, but then $G_{n}=H_{n}$, which is a contradiction. Thus $K \subset G$.

Now $H_{n} G_{n+1}=G_{n}$ and $H \subset K$ imply that $K_{n} G_{n+1}=G_{n}$, therefore $K_{n}=G_{n}$ since otherwise the lemma is false for $K$, a larger subgroup than $H$. But also $K_{n+1} \subset G_{n+1}$, since if $K_{n+1}=G_{n+1}$, then $H_{n} K_{n+1}=G_{n}$ $=K_{n}$ while $H_{n} \subset G_{n}=K_{n}$, and the lemma is false for $K$, a group of smaller order than $G$. Moreover, $K_{n+1} G_{n+2} \subset G_{n+1}$, since otherwise $K$ would be a subgroup larger than $H$ for which the lemma is false (in this case for $m=n+1$ ).

Clearly $H_{n+1} G_{n+2}$ is normal in $G$. Also, $\left(G_{n}, H\right)=\left(H_{n} \times N, H\right)=H_{n+1}$ since $N$ is in the center of $G$, hence $\left(G_{n}, H\right) \subseteq H_{n+1} G_{n+2}$. Thus, by Lemma $1,\left(G, H_{n}\right) \subseteq H_{n+1} G_{n+2}$. But $G_{n+1}=\left(G_{n}, G\right)=\left(H_{n} \times N, G\right)=\left(H_{n}, G\right)$, so $G_{n+1} \subseteq H_{n+1} G_{n+2} \subseteq K_{n+1} G_{n+2} \subset G_{n+1}$. This is a contradiction, and the lemma follows.

Proof of Theorem 1. We assume $G$ is of minimal order and $H$ is a subgroup of $G$ having maximal order such that the theorem is false. If $K$ is a subgroup of $G$ such that $G \supset K \supset H$, then $K_{n}=G_{n}$. For if $K_{n} \subset G_{n}$, it follows from the maximality of $H$ that $(K \phi(G))_{n} \subset G_{n}$, and consequently $(H \phi(G))_{n} \subset G_{n}$. Moreover, $(H \phi(K))_{n} \subset K_{n}$. For if $(H \phi(K))_{n}=K_{n}$, then since $H_{n} \subset G_{n}=K_{n}, K$ is a smaller group than $G$ for which the theorem is false. Thus $H \phi(K) \subseteq H$, and hence $\phi(K) \subseteq H$. Now also $\left(H G_{2}\right)_{n} \subseteq H_{n} G_{n+1}$, so that by Lemma $2\left(H G_{2}\right)_{n} \subset G_{n}$. Hence, $H G_{2} \subseteq H$ and $H$ is normal in $G$.

If $\langle x, H\rangle \subset G$ for every $x$ in $G$, then $x^{p} \in \phi(\langle x, H\rangle) \subseteq H$ for every $x$ in $G$. Thus in this case $P(G) \subseteq H$. But $G_{2} \subseteq H$, so that $\phi(G) \subseteq H$ which is impossible.

Thus there is an $x$ such that $\langle x, H\rangle=G$. Since $x^{p} \in \phi(G),\left\langle x^{p}, H\right\rangle \subset G$. But then $x^{p^{2}} \in \phi\left(\left\langle x^{p}, H\right\rangle\right) \subseteq H$, so that $H$ has index at most $p^{2}$ in $G$. 
Since $G$ is noncyclic, $\phi(G)$ is the intersection of all normal subgroups of index $p^{2}$ in $G$; hence $\phi(G) \subseteq H$ which is impossible.

The theorem is thus proved.

CoRollary. If $G$ is a finite p-group which can be generated by two elements, and if $G_{n} \neq\langle 1\rangle$, then $H_{n} \subset G_{n}$ for every proper subgroup $H$ of $G$.

Proof. If $H \subset G$ there exists a subgroup $N$ of $G$ such that $[G: N]=p$ and $H \subseteq N$. Then $\phi(G) \subseteq N$, and since $G$ can be generated by two elements, there exists an element $x$ such that $N=\langle x, \phi(G)\rangle$. The corollary is trivial if $n=1$. But if $n \geqq 2$ then $\langle x\rangle_{n}=1$, so that by Theorem 1 , $N_{n}=(\langle x\rangle \phi(G))_{n} \subset G_{n}$. The corollary follows, since $H_{n} \subseteq N_{n}$.

As another application of Lemmas 1 and 2 we prove the following.

THEOREM 2. If $G$ is a finite, nilpotent group and $H$ is a subgroup of $G$ such that $H_{n}=G_{n}$, then $H_{n+k}=G_{n+k}$ for every positive integer $k$.

Proof. It will suffice to prove the theorem for $G$ a finite $p$-group. We prove that $H_{n+1}=G_{n+1}$. Note that since $\left(H G_{2}\right)_{n+1} \subseteq H_{n+1} G_{n+2}$, it would follow from $H_{n+1} \subset G_{n+1}$ and Lemma 2 that $\left(H G_{2}\right)_{n+1} \subset G_{n+1}$. Thus if the theorem fails it must fail for a normal subgroup $H$. But using Lemma 1 we have

$$
G_{n+1}=\left(G, G_{n}\right)=\left(G, H_{n}\right) \subseteq\left(G_{n}, H\right)=\left(H_{n}, H\right)=H_{n+1}
$$

if $H$ is normal. Thus the theorem is proved.

\section{REFERENCES}

1. M. Hall, The theory of groups, New York, The Macmillan Company, 1959.

2. N. Ito, On a theorem of L. Rédei and J. Szêp concerning p-groups, Acta. Univ. Szeged. vol. 14 (1952) pp. 186-187.

California Institute of Technology 